\title{
Constraints on non-standard flavor-dependent interactions from Superkamiokande and Hyperkamiokande
}

\author{
OSAMU YASUDA* \\ Department of Physics, Tokyo Metropolitan University \\ Hachioji, Tokyo 192-0397, Japan \\ E-mail: vasuda AT phys.se.tmu.ac.ip
}

\begin{abstract}
We investigate the constraint on the flavor-dependent neutral current Non-Standard Interactions in propagation from atmospheric neutrino experiments Superkamiokande and Hyperkamiokande. With the ansatz where the parameters which have strong constraints from other experiments are neglected, we show how these experiments put constraints on the remaining parameters of the Non-Standard Interactions.
\end{abstract}

16th International Workshop on Neutrino Factories and Future Neutrino Beam Facilities 25 -30 August, 2014

University of Glasgow, United Kingdom

\footnotetext{
* Speaker.
} 


\section{Introduction}

The phenomenon of neutrino oscillation is described by the mixing matrix

$$
U=\left(\begin{array}{ccc}
c_{12} c_{13} & s_{12} c_{13} & s_{13} e^{-i \delta} \\
-s_{12} c_{23}-c_{12} s_{23} s_{13} e^{i \delta} & c_{12} c_{23}-s_{12} s_{23} s_{13} e^{i \delta} & s_{23} c_{13} \\
s_{12} s_{23}-c_{12} c_{23} s_{13} e^{i \delta} & -c_{12} s_{23}-s_{12} c_{23} s_{13} e^{i \delta} & c_{23} c_{13}
\end{array}\right),
$$

where $s_{j k} \equiv \sin \theta_{j k}$ and $c_{j k} \equiv \cos \theta_{j k}, \theta_{j k}$ with $(j, k)=(1,2),(1,3),(2,3)$ are the three mixing angles and $\delta$ is the Dirac CP phase. Thanks to recent neutrino experiments [ $[$ ], all the mixing angles and the mass squared differences have been measured, and the only unknown quantities which can probed by neutrino oscillation are the mass hierarchy pattern and $\delta$. It is believed that these unknown quantities will be determined in the future neutrino experiments, including those with intense accelerator neutrino beams [ [2, B]]. It is also expected that these future experiments with intense accelerator neutrino beams will enable us to probe new physics beyond the standard model with massive neutrinos, by looking for the deviation from the standard scenario.

In the standard model with three massive neutrinos, the Dirac equation for the flavor eigenstate $\Psi^{T} \equiv\left(v_{e}, v_{\mu}, v_{\tau}\right)$ of neutrino in matter is given by

$$
i \frac{d \Psi}{d t}=\left[U \operatorname{diag}\left(E_{1}, E_{2}, E_{3}\right) U^{-1}+\mathscr{A}\right] \Psi
$$

where the matter potential is given by

$$
\mathscr{A}=A\left(\begin{array}{lll}
1 & 0 & 0 \\
0 & 0 & 0 \\
0 & 0 & 0
\end{array}\right) \text {. }
$$

Here $A \equiv \sqrt{2} G_{F} n_{e}$ stands for the magnitude of the standard matter effect due to the charged current interaction, $n_{e}$ is the number density of the electron in the matter, and the matter effect due to the neutral current interaction, which is proportional to the unit matrix in the flavor basis, is ignored because it would affect only the phase of the oscillation probability amplitude.

Here I would like to consider the flavor-dependent nonstandard four-fermi interactions

$$
\mathscr{L}_{\mathrm{eff}}^{\mathrm{NSI}}=-2 \sqrt{2} \varepsilon_{\alpha \beta}^{f P} G_{F}\left(\bar{v}_{\alpha} \gamma_{\mu} P_{L} v_{\beta}\right)\left(\bar{f} \gamma^{\mu} P f\right),
$$

where only the interactions with $f=e, u, d$ are relevant to the flavor transition of neutrino due to the matter effect, $G_{F}$ denotes the Fermi coupling constant, $P$ stands for a projection operator and is either $P_{L} \equiv\left(1-\gamma_{5}\right) / 2$ or $P_{R} \equiv\left(1+\gamma_{5}\right) / 2$. (때) is the most general form of the interactions which conserve electric charge, color, and lepton number [四]. In the presence of these interactions ([L.3), the matter potential is modified to

$$
\mathscr{A} \rightarrow A\left(\begin{array}{ccc}
1+\varepsilon_{e e} & \varepsilon_{e \mu} & \varepsilon_{e \tau} \\
\varepsilon_{e \mu}^{*} & \varepsilon_{\mu \mu} & \varepsilon_{\mu \tau} \\
\varepsilon_{e \tau}^{*} & \varepsilon_{\mu \tau}^{*} & \varepsilon_{\tau \tau}
\end{array}\right)
$$

where $\varepsilon_{\alpha \beta}$ are defined as $\varepsilon_{\alpha \beta} \equiv \sum_{f, P}\left(n_{f} / n_{e}\right) \varepsilon_{\alpha \beta}^{f P} \simeq \sum_{P}\left(\varepsilon_{\alpha \beta}^{e P}+3 \varepsilon_{\alpha \beta}^{u P}+3 \varepsilon_{\alpha \beta}^{d P}\right)$, $n_{f}$ is the number density of $f$ in matter, and we have taken into account the fact that the number density of $u$ quarks 
and $d$ quarks are three times as that of electrons. The constraint on $\varepsilon_{\alpha \beta}$ haven been given by a number of works, and can be summarized as [5]

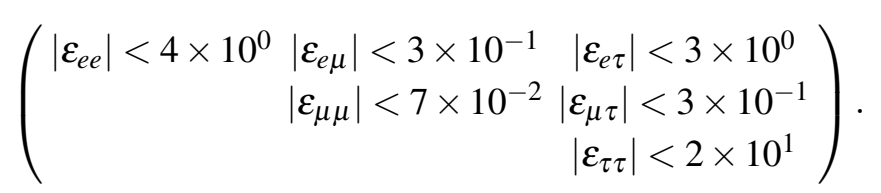

From Eq. ([ᄄ.5) we see that

$$
\varepsilon_{e \mu} \simeq \varepsilon_{\mu \mu} \simeq \varepsilon_{\mu \tau} \simeq 0
$$

is satisfied.

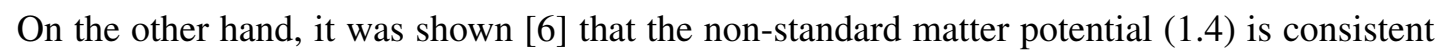
with the high energy atmospheric neutrino data only if

$$
\varepsilon_{\tau \tau} \simeq \frac{\left|\varepsilon_{e \tau}\right|^{2}}{1+\varepsilon_{e e}}
$$

is satisfied.

In this talk I will discuss the constraints on the flavor-dependent non-standard interactions from the Superkamiokande atmospheric neutrino data. Taking the constraints $(\mathbb{L C})$ and $(\mathbb{L} . \mathbb{Z})$ into consideration, for simplicity I will take the following ansatz for the matter potential ${ }^{1}$ :

$$
A\left(\begin{array}{ccc}
1+\varepsilon_{e e} & 0 & \varepsilon_{e \tau} \\
0 & 0 & 0 \\
\varepsilon_{e \tau}^{*} & 0 & \frac{\left|\varepsilon_{e} \tau\right|^{2}}{1+\varepsilon_{e e}}
\end{array}\right)
$$

I will also give the expected sensitivity to the same parameters from the future atmospheric neutrino data by the Hyperkamiokande experiment [ए]]. The result will be given as the allowed region in the $\left(\varepsilon_{e e},\left|\varepsilon_{e \tau}\right|\right)$ plane by marginalizing the $\chi^{2}$ with respect to the standard oscillation parameters as well as $\arg \left(\varepsilon_{e \tau}\right)$.

\section{Analysis}

The data we analyzed is for 3903 days [U]]. The analysis [ㅁ] by the Superkamiokande collaboration uses information which is even more detailed than the one in Ref. [12], and we have been unable to reproduce their results of the Monte Carlo simulation. So we have combined the two sub-GeV $\mu$-like data set in one, the two multi-GeV e-like in one, the two partially contained event data set and the multi-GeV $\mu$-like in one, and the three upward going $\mu$ in one. The analysis was performed with the code which were used in Refs. [ㅍ, 때, 피]. $\chi^{2}$ is defined as

$$
\chi^{2}=\min _{\theta_{23},\left|\Delta m_{32}^{2}\right|, \delta, \arg \left(\varepsilon_{e \tau}\right)}\left[\chi_{\text {sub-GeV }}^{2}+\chi_{\text {multi-GeV }}^{2}+\chi_{\text {upward }}^{2}\right],
$$

\footnotetext{
${ }^{1}$ The constraints from the atmospheric neutrino has been discussed in Refs [ [ $\left.\mathbb{Q}, \mathbb{Q}, \mathbf{Q}\right]$ ] with the ansatz different from ours
} 
where

$$
\begin{aligned}
\chi_{\text {sub }-\mathrm{GeV}}^{2}= & \min _{\alpha, \beta^{\prime} s}\left[\frac{\beta_{s 1}^{2}}{\sigma_{\beta s 1}^{2}}+\frac{\beta_{s 2}^{2}}{\sigma_{\beta s 2}^{2}}\right. \\
& +\sum_{j=1}^{10}\left\{\frac{1}{n_{j}^{\mathrm{s}}(e)}\left[\alpha\left(1-\frac{\beta_{s 1}}{2}+\frac{\beta_{s 2}}{2}\right) N_{j}^{\mathrm{s}}(e)+\alpha\left(1-\frac{\beta_{s 1}}{2}-\frac{\beta_{s 2}}{2}\right) \bar{N}_{j}^{\mathrm{s}}(e)-n_{j}^{\mathrm{s}}(e)\right]^{2}\right. \\
& \left.\left.+\frac{1}{n_{j}^{\mathrm{s}}(\mu)}\left[\alpha\left(1+\frac{\beta_{s 1}}{2}+\frac{\beta_{s 2}}{2}\right) N_{j}^{\mathrm{s}}(\mu)+\alpha\left(1+\frac{\beta_{s 1}}{2}-\frac{\beta_{s 2}}{2}\right) \bar{N}_{j}^{\mathrm{s}}(\mu)-n_{j}^{\mathrm{s}}(\mu)\right]^{2}\right\}\right], \\
\chi_{\mathrm{multi}-\mathrm{GeV}}^{2}= & \min _{\alpha, \beta^{\prime} s}\left[\frac{\beta_{m 1}^{2}}{\sigma_{\beta m 1}^{2}}+\frac{\beta_{m 2}^{2}}{\sigma_{\beta m 2}^{2}}\right. \\
& +\sum_{j=1}^{10}\left\{\frac{1}{n_{j}^{\mathrm{m}}(e)}\left[\alpha\left(1-\frac{\beta_{m 1}}{2}+\frac{\beta_{m 2}}{2}\right) N_{j}^{\mathrm{m}}(e)+\alpha\left(1-\frac{\beta_{m 1}}{2}-\frac{\beta_{m 2}}{2}\right) \bar{N}_{j}^{\mathrm{m}}(e)-n_{j}^{\mathrm{m}}(e)\right]^{2}\right. \\
& \left.+\frac{1}{n_{j}^{\mathrm{m}}(\mu)}\left[\alpha\left(1+\frac{\beta_{m 1}}{2}+\frac{\beta_{m 2}}{2}\right) N_{j}^{\mathrm{m}}(\mu)+\alpha\left(1+\frac{\beta_{m 1}}{2}-\frac{\beta_{m 2}}{2}\right) \bar{N}_{j}^{\mathrm{m}}(\mu)-n_{j}^{\mathrm{m}}(\mu)\right]^{2}\right\}, \\
\chi_{\text {upward }}^{2}= & \min _{\alpha}\left[\frac{\alpha^{2}}{\sigma_{\alpha}^{2}}+\sum_{j=1}^{10} \frac{1}{n_{j}^{\mathrm{u}}(\mu)}\left[\alpha N_{j}^{\mathrm{u}}(\mu)-n_{j}^{\mathrm{u}}(\mu)\right]^{2}\right] .
\end{aligned}
$$

are $\chi^{2}$ for the sub-GeV, multi-GeV, and upward going $\mu$ events, respectively, the summation on $j$ runs over the ten zenith angle bins for each $\chi^{2}, N_{j}^{a}(\alpha)$ and $n_{j}^{a}(\alpha)(a=\mathrm{s}, \mathrm{m}, \mathrm{u} ; \alpha=\mathrm{e}, \mu)$ stand for the theoretical predictions and data for the numbers of the sub-GeV, multi-GeV, and upward going $\mu$ events, and it is understood that $\chi^{2}$ is minimized with respect to all the normalization factors $\alpha$, $\beta_{s}, \beta_{m}$. We have put $\sigma_{s 1}=\sigma_{m 1}=0.03, \sigma_{s 2}=\sigma_{m 2}=0.05, \sigma_{\alpha}=0.2$ and we have assumed that the overall flux normalization $\alpha$ in the contained events is a free parameter as in [प्D], and we have omitted the other uncertainties, such as the $E_{v}$ spectral index, the relative normalization between $\mathrm{PC}$ and FC and up-down correlation, etc., for simplicity.

In Eq. (‥J) the sum of each $\chi^{2}$ is optimized with respect the mixing angle $\theta_{23}$, the mass squared difference $\left|\Delta m_{32}^{2}\right|$, the Dirac CP phase $\delta$ and the phase $\arg \left(\varepsilon_{e \tau}\right)$ of the parameter $\varepsilon_{e \tau}$. The other oscillation parameters give little effect on $\chi^{2}$, so we have fixed them as $\sin ^{2} 2 \theta_{12}=0.86$, $\sin ^{2} 2 \theta_{13}=0.1$ and $\Delta m_{21}^{2}=7.6 \times 10^{-5} \mathrm{eV}^{2}$.

The result for the Superkamiokande data for 3903 days is given in Fig. $\mathrm{W}$. The best-fit point is $\varepsilon_{e e}=-1,\left|\varepsilon_{e \tau}\right|=0$, and the value of $\chi^{2}$ at this point is 74.7 (74.5) for 50 degrees of freedom in the case of the normal (inverted) hierarchy, and goodness of fit is $2.5 \sigma \mathrm{CL}$ in both cases. The best-fit point is different from the standard case $\varepsilon_{e e}=\left|\varepsilon_{e \tau}\right|=0$, and this may be because we have been unable to reproduce the Monte Carlo simulation by the Superkamiokande group. The difference of the value of $\chi^{2}$ for the standard case and that for the best-fit point is $\Delta \chi^{2}=2.6$ (2.0) for 2 degrees of freedom in the case of the normal (inverted) hierarchy, and its significance is 1.1 (0.9) $\sigma \mathrm{CL}$. So the standard case is certainly acceptable in our analysis. From the Fig. W we observe that the allowed region for $|\tan \beta| \equiv\left|\varepsilon_{e \tau}\right| /\left|1+\varepsilon_{e e}\right|$ is approximately $|\tan \beta| \equiv\left|\varepsilon_{e \tau}\right| /\left|1+\varepsilon_{e e}\right| \lesssim 0.8$ at $2.5 \sigma \mathrm{CL}$.

We have also performed the analysis for the Hyperkamiokande case with the same period of time, i.e., for 3903 days. In this case we have assumed that the data $n_{j}^{a}(\alpha)(a=\mathrm{s}, \mathrm{m}, \mathrm{u} ; \alpha=\mathrm{e}, \mu)$ are 

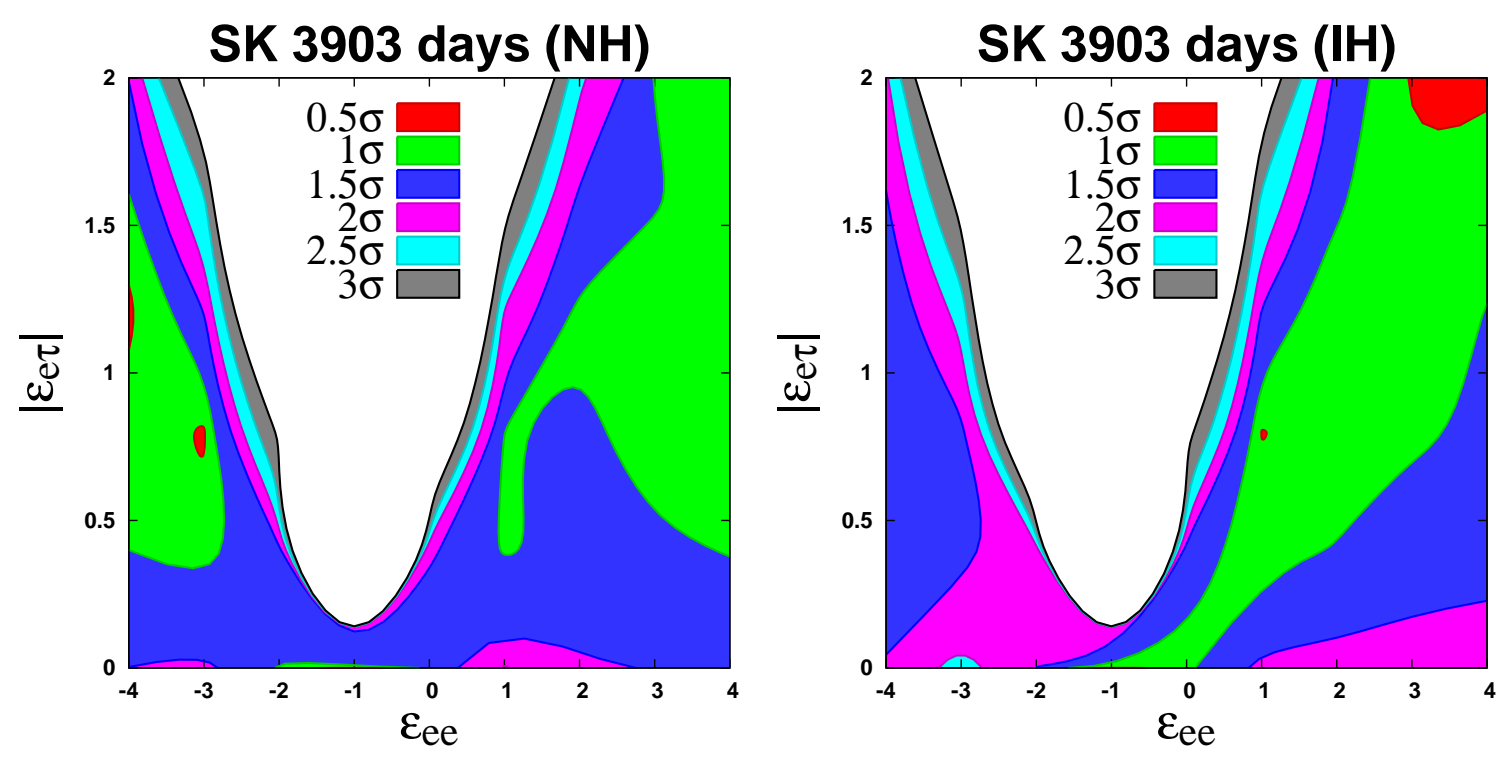

Figure 1: The allowed region in the $\left(\varepsilon_{e e},\left|\varepsilon_{e \tau}\right|\right)$ plane of the Superkamiokande atmospheric neutrino data for 3903 days for the Normal Hierarchy (left panel) and the Inverted Hierarchy (right panel).

the numbers of events which are expected from the standard oscillation scenario with parameters $\theta_{23}=\pi / 4,\left|\Delta m_{32}^{2}\right|=2.5 \times 10^{-3} \mathrm{eV}^{2}$. We have further assumed for simplicity that $\delta=0$. The result for the Hyperkamiokande case is given in Fig. [2. In this case the allowed region for $|\tan \beta| \equiv$ $\left|\varepsilon_{e \tau}\right| /\left|1+\varepsilon_{e e}\right|$ is approximately $|\tan \beta| \equiv\left|\varepsilon_{e \tau}\right| /\left|1+\varepsilon_{e e}\right| \lesssim 0.4$ at $2.5 \sigma \mathrm{CL}$.

\section{Conclusion}

In this talk I have shown that the flavor-dependent neutral current Non-Standard Interactions in propagation can be constrained from the atmospheric neutrino data by Superkamiokande and Hyperkamiokande. With the ansatz ([.8) for the parameters of the Non-Standard Interactions, I have shown the allowed region in the $\left(\varepsilon_{e e},\left|\varepsilon_{e \tau}\right|\right)$ plane. In the case of the Superkamiokande data for 3903 days, the allowed region is described by $|\tan \beta| \equiv\left|\varepsilon_{e \tau}\right| /\left|1+\varepsilon_{e e}\right| \lesssim 0.8$ at $2.5 \sigma \mathrm{CL}$, while it is given by $|\tan \beta| \equiv\left|\varepsilon_{e \tau}\right| /\left|1+\varepsilon_{e e}\right| \lesssim 0.4$ at $2.5 \sigma \mathrm{CL}$ in the Hyperkamiokande case.

\section{References}

[1] K. A. Olive et al. [Particle Data Group Collaboration], Chin. Phys. C 38 (2014) 090001.

[2] K. Abe et al. [Hyper-Kamiokande Working Group Collaboration], arXiv:1412.4673 [physics.ins-det].

[3] C. Adams et al. [LBNE Collaboration], arXiv:1307.7335 [hep-ex].

[4] S. Davidson, C. Pena-Garay, N. Rius and A. Santamaria, JHEP 0303, 011 (2003) [arXiv:hep-ph/0302093].

[5] C. Biggio, M. Blennow and E. Fernandez-Martinez, JHEP 0908 (2009) 090 [arXiv:0907.0097 [hep-ph]]. 

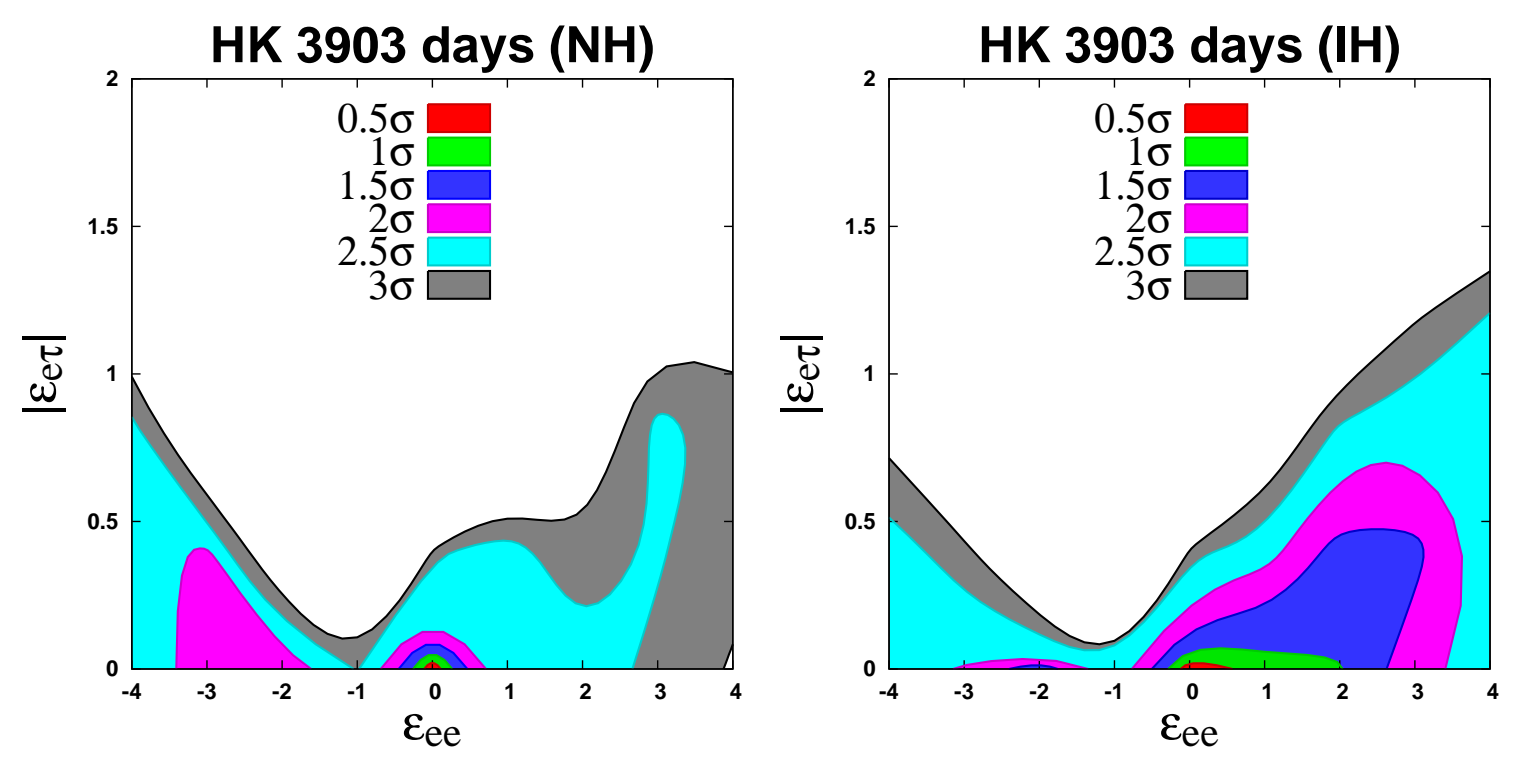

Figure 2: The expected allowed region in the $\left(\varepsilon_{e e},\left|\varepsilon_{e \tau}\right|\right)$ plane of the Hyperkamiokande atmospheric neutrino data for 3903 days for the Normal Hierarchy (left panel) and the Inverted Hierarchy (right panel). The best-fit point is the standard case with $\varepsilon_{e e}=\left|\varepsilon_{e \tau}\right|=0$.

[6] A. Friedland and C. Lunardini, Phys. Rev. D 72 (2005) 053009 [hep-ph/0506143].

[7] M. C. Gonzalez-Garcia, M. Maltoni and J. Salvado, JHEP 1105 (2011) 075 [arXiv:1103.4365 [hep-ph]].

[8] G. Mitsuka et al. [Super-Kamiokande Collaboration], Phys. Rev. D 84 (2011) 113008 [arXiv:1109.1889 [hep-ex]].

[9] M. C. Gonzalez-Garcia and M. Maltoni, JHEP 1309 (2013) 152 [arXiv:1307.3092].

[10] K. Abe, T. Abe, H. Aihara, Y. Fukuda, Y. Hayato, K. Huang, A. K. Ichikawa and M. Ikeda et al., arXiv:1109.3262 [hep-ex].

[11] Y. Itow, Nucl. Phys. Proc. Suppl. 235-236 (2013) 79.

[12] Y. Ashie et al. [Super-Kamiokande Collaboration], Phys. Rev. D 71 (2005) 112005 [hep-ex/0501064].

[13] R. Foot, R. R. Volkas and O. Yasuda, Phys. Rev. D 58 (1998) 013006 [hep-ph/9801431].

[14] O. Yasuda, Phys. Rev. D 58 (1998) 091301 [hep-ph/9804400].

[15] O. Yasuda, hep-ph/0006319. 